\title{
Duodenal Cancer Diagnosed by Capsule Endoscopy and Double Balloon Enteroscopy in a Patient with Hereditary Nonpolyposis Colorectal Cancer
}

\author{
Hirokazu Yamagami Nobuhide Oshitani Noriko Kamata Mitsue Sogawa \\ Kenji Watanabe Kazuhide Higuchi Tetsuo Arakawa \\ Department of Gastroenterology, Osaka City University Graduate School of Medicine, Abeno-ku, Osaka, Japan
}

Dear Sir,

Hereditary nonpolyposis colorectal cancer (HNPCC) involves a predisposition not only to colorectal cancer, but also to cancers of various other organs (the endometrium, stomach, ovaries, small bowel, hepatobiliary epithelium, uroepithelia epithelium and brain) [1]. However, the diagnosis of small bowel cancer without a laparotomy was difficult. Here we report the case of duodenal cancer diagnosed by capsule endoscopy (CE) and double balloon enteroscopy (DBE) in a patient with HNPCC.

The patient is a 72-year-old woman. She complained of passing tarry stool and was admitted to our hospital for further examination. History revealed that she had undergone operations for advanced rectal cancer at age 33 and for another advanced colon cancer at ages 49 and 51. Additionally, she had undergone operations for uterine cancer at the age of 50 . Her mother died of colon cancer at age 49; 3 of her 4 siblings were affected by colorectal


Fig. 1. a Capsule endoscopic appearance showing intestinal stenosis in the third part of the duodenum. b Double balloon enteroscopic appearance showed advanced cancer in the third part of the duodenum. Biopsy was taken and a well-differentiated tubular adenocarcinoma was diagnosed.

\section{KARGER}

Fax +41613061234 E-Mail karger@karger.ch www.karger.com
Hirokazu Yamagami

Department of Gastroenterology, Osaka City University Graduate School of Medicine 1-4-3 Asahi-machi, Abeno-ku

Osaka 545-8585 (Japan)

Tel. +81 66645 3811, Fax +81 66645 3813, E-Mail yamagami@med.osaka-cu.ac.jp 
cancer, and her sister and daughter were affected by uterine cancer. Her pedigree included 4 patients with advanced colon cancer and 2 with uterine cancer. Therefore, a diagnosis of HNPCC was made, according to the clinical criteria based on her past and family histories.

Esophagogastroduodenoscopy demonstrated no evidence of gastrointestinal bleeding. We performed a CE to identify the origin of the bleeding. CE revealed stenosis due to tumor with hemorrhage at the third portion of the duodenum (fig. 1a). DBE revealed a duodenal tumor (fig. 1b), confirmed on biopsy to be a well-differentiated tubular adenocarcinoma. A pancreatoduodenectomy was performed. Histologically, the lesion was a moderately differentiated adenocarcinoma of the duodenum.

\section{Discussion}

The most frequent causes of death in HNPCC patients are colorectal cancer, endometrial cancer, brain tumor, lung cancer, and gastric cancer. A significant decrease in the standardized mortality ratio (SMR) for colorectal cancer was observed in a large cohort in the period 1990-2004 compared with the period 1960-1975 owing to distribution of colonoscopic surveillance. However, over all periods, the SMR in HNPCC patients was significantly increased for cancer of the small bowel, brain, kidney, ovaries, pancreas, and stomach. Among all tumors other than colorectal and endometrial cancers, the SMR is the highest in small bowel cancer [2] Therefore, an effective screening program is required for the detection of colorectal and extracolonic cancers in HNPCC patients.

$\mathrm{CE}$ has been established as an effective diagnostic modality to diagnose small bowel tumors [3]. CE is appropriate for first inspection and DBE is suitable for further examination that will enable treatment and histopathological diagnosis.

In our case, CE and DBE were useful for detecting small bowel lesions in a HNPCC patient. Small bowel surveillance with $\mathrm{CE}$ should be useful in the diagnosis of HNPCC-associated small bowel cancer; however, the frequency of a CE surveillance program needs to be considered.

\section{References}

1 Watson P, Lynch HT: Extracolonic cancer in hereditary nonpolyposis colorectal cancer. Cancer 1993;71:677-685.

2 de Jong AE, Hendriks YM, Kleibeuker JH, de Boer SY, Cats A, Griffioen G, Nagengast FM, et al: Decrease in mortality in Lynch syndrome families because of surveillance. Gastroenterology 2006;130:665-671.

3 Cobrin GM, Pittman RH, Lewis BS: Increased diagnostic yield of small bowel tumors with capsule endoscopy. Cancer 2006; 107:22-27.

\section{Announcement}

\section{1st Pan American Congress of Digestive Diseases Global Challenges in Gastroenterology from the End of the World}

Santiago, Chile, November 11-14, 2008 